

\title{
Some Aspects of Analogy in Mathematical Reasoning
}

Manfred Kerber

Published as: In Klaus P. Jantke, ed., Analogical and Inductive Inference; International Workshop AII'89, Reinhardsbrunn Castle, GDR, p.231-242, Springer Verlag, Berlin, Germany, LNAI 397, 1989. 


\title{
Some Aspects of Analogy in Mathematical Reasoning
}

\author{
Manfred Kerber \\ Fachbereich Informatik, Universität Kaiserslautern \\ D-6750 Kaiserslautern, West Germany \\ UUCP: ....!mcvax!unido!uklirb!kerber
}

\begin{abstract}
An important research problem is the incorporation of "declarative" knowledge into an automated theorem prover that can be utilized in the search for a proof. An interesting proposal in this direction is Alan Bundy's approach of using explicit proof plans that encapsulate the general form of a proof and is instantiated into a particular proof for the case at hand. We give some examples that show how a "declarative" highlevel description of a proof can be used to find proofs of apparently "similiar" theorems by analogy. This "analogical" information is used to select the appropriate axioms from the database so that the theorem can be proved. This information is also used to adjust some options of a resolution theorem prover. In order to get a powerful tool it is necessary to develop an epistemologically appropriate language to describe proofs, for which a large set of examples should be used as a testbed. We present some ideas in this direction.
\end{abstract}

Keywords: analogy, theorem proving, proof plans, abstract description

... et chaque vérité que je trouvais étant une règle qui me servait après à en trouver d'autres, ...

René Descartes, Discours de la Méthode

\section{Introduction}

Analogy plays an important rôle in mathematics: a main part of the formation of concepts as well as of general reasoning is done by analogy. Mathematicians know many concepts, theorems, and examples and out of this fund they can invent new concepts, find new theorems, construct new proofs, and enlarge this fund of examples. George Pólya writes in [15] that a proof is not completed once it is found and written down, but then perhaps the most important steps are to analyze the proof and to learn from it for the solution of future problems. Finding the solution of a problem often consists in reformulating it into a problem that is analogous to a solved one. In [16] Pólya writes that two systems are analogous, if they agree in clearly definable relations of 
their respective parts. As examples he mentions a geometrical analogy, namely a triangle in the plane and a tetrahedron in the space, and an analytical example of determining the sums of infinite series. PólyA's ideas are not formalized as his purpose is to teach how to instruct students in mathematics. Instead of a formalization he presents many examples, by means of which a pupil can learn also for other cases. But as pointed out by Allen Newell [14] there is a considerable gap between the advice Pólya gives and what can be used directely in a computer system, because - if one wants to use PÓLYA's heuristic - one has to master "wild subproblems" as NEWELL calls them, that is, it is still a problem to utilize the solution of a "subproblem" to the solution of the proper problem.

In the following we consider only some simple aspects of analogy and give examples how the utilization of such analogies can be profitable. We do not give a formalization of analogy, but only present some ideas, how certain aspects might be formalized. In general it seems that "analogy" is a term that stands for many methods and techniques with some common features, just as for example in "machine learning", where almost certainly no single method will be sufficient for all its aspects. However we argue that even the simple aspects that are proposed here can lead to drastic improvements when they are appropriately used in an automated theorem prover. Our method of using analogy does not enlarge the possibilities of deduction in principle, but it is helpful in guiding the search for a proof. In addition we give some hints how it might be possible to find the preconditions that are necessary for the proof of a theorem.

Most examples are taken from [5], as the idea to look for analogies in proofs arose in JörG SIEKMANN's project, where many of the theorems of a textbook on automata theory [5] where proved with the help of the MKRP theorem proving system [6, 12].

Many different approaches exist to capture certain aspects of analogy:

- Pairings of or mappings between the analogous parts

- Abstraction to a common generalization

- Relation between the signatures based on a model-theoretical approach

- Proof plans to find proofs in a certain domain

We are not going to give an overview on all these efforts. We refer to the overview article of RoGERS P. HALL [11]. The idea presented in this paper is closely related to proof plans, that is, to describe the proofs abstractely. If a proof has been found the plan should be analyzed and simplified. Then - so the hope - this proof plan can be used to find proofs of similiar theorems. On the importance of abstract description of theorems and proofs see also [8].

\section{Thesis}

To find an analogy depends, just as many other intelligent processes, to a great degree on finding the adequate level of abstraction. When we are searching for an analogy we are taking only certain aspects of the situation into account and are disregarding others. HeLmut ThiELE [18] uses subsystems of the signature to abstract from unimportant parts of a concept, in other words to neglect those parts that are not necessary when only the two analogous facts are taken into account. The remaining important parts then have to be isomorphic. In some sense this can be seen as an equivalence mapping among models. In mathematics we can follow a similiar simple approach by not telling the system the meaning, that is, the definition of some concept. For example if we want to consider only the "reflexivity" of an equivalence relation, we abstract from "symmetry" 
and "transitivity". In other words we want to model reasoning of the sort "whatever symmetry and transitivity might be, we can conclude ..." (see the example below). This method is well known in the field of automated theorem proving, and is in fact the second "cheat" of ALAN BUNDY'S (originally longer) list in [3, p.88]:

- "Feeding to the theorem prover only those axioms known to be required in the proof. Irrelevant axioms can dramatically increase the size of the search tree."

- "Generating only that part of the search tree which lies within some arbitrary limits, but which is known to include the proof. Typically, the sought proof is examined to see: how long it is; what the maximum depth of function nesting is and what the maximum length of clauses is: and then the tree is only searched within those limits."

We give examples how "analogy" can help at least in some cases to automate the finding of those clauses that are necessary to derive a theorem and to automatically adjust some "options" of a resolution prover in order to cut down the search space. Unlike other approaches of using analogy as that of GREINER [9], where facts that are neither in the data base nor logically derivable, can be derived by analogy, our treatment of analogy does not enlarge the derivation facilities in principle.

Additionally we argue that the description level of concepts and theorems should be as high as possible, as it is much easier then to find the corresponding level of abstraction where the analogy can be expressed. If the level is too high one can expand the definitions stepwise without any problem, the reverse operation is much more difficult.

\section{Main Example}

In this part we analyze the following simple theorem in detail (taken from [5, p.37]):

Theorem: Let $S$ be a set and $\rho$ and $\sigma$ be two equivalence relations on $S$, then the relation $\rho \cap \sigma$ is also an equivalence relation.

If we use a sorted first order logic to formalize the theorem and prove it automatically by a theorem prover as the MKRP system, we may axiomatize it in the following way:

- Definition of an equivalence relation:

$\forall \rho$ :relation equivalence_relation $(\rho) \Longleftrightarrow$ reflexive $(\rho) \wedge$ symmetric $(\rho) \wedge \operatorname{transitive}(\rho)$

- Definition of reflexivity:

$\forall \rho$ : relation reflexive $(\rho) \Longleftrightarrow(\forall x: S(x, x) \in \rho)$

- Definition of symmetry:

$\forall \rho$ : relation symmetric $(\rho) \Longleftrightarrow(\forall x, y: S \quad(x, y) \in \rho \Longrightarrow(y, x) \in \rho)$

- Definition of transitivity:

$\forall \rho$ : relation transitive $(\rho) \Longleftrightarrow(\forall x, y, z: S \quad(x, y) \in \rho \wedge(y, z) \in \rho \Longrightarrow(x, z) \in \rho)$

- Definition of intersection:

$\forall \rho, \sigma$ : relation $\forall x, y: S(x, y) \in \rho \cap \sigma \Longleftrightarrow(x, y) \in \rho \wedge(x, y) \in \sigma$

- Theorem:

$\forall \rho, \sigma$ :relation equivalence_relation $(\rho) \wedge$ equivalence_relation $(\sigma)$

$\Longrightarrow$ equivalence_relation $(\rho \cap \sigma)$ 
Let us use the following abbreviation:

$$
\begin{aligned}
P(\rho, \sigma) \Longleftrightarrow \quad & \text { reflexive }(\rho) \wedge \operatorname{symmetric}(\rho) \wedge \operatorname{transitive}(\rho) \wedge \\
& \text { reflexive }(\sigma) \wedge \operatorname{symmetric}(\sigma) \wedge \operatorname{transitive}(\sigma)
\end{aligned}
$$

Expanding the definition of an equivalence relation the theorem can be rewritten as:

$\forall \rho, \sigma:$ relation $P(\rho, \sigma) \Longrightarrow$ reflexive $(\rho \cap \sigma) \wedge \operatorname{symmetric}(\rho \cap \sigma) \wedge \operatorname{transitive}(\rho \cap \sigma)$

We (as well as the MKRP system) see that one can split the proof into the three subproofs:

- $\forall \rho, \sigma$ : relation $P(\rho, \sigma) \Longrightarrow$ reflexive $(\rho \cap \sigma)$

- $\forall \rho, \sigma$ : relation $P(\rho, \sigma) \Longrightarrow \operatorname{symmetric}(\rho \cap \sigma)$

- $\forall \rho, \sigma:$ relation $P(\rho, \sigma) \Longrightarrow$ transitive $(\rho \cap \sigma)$

These subproofs are independent, so the order of the subproofs is a priori irrelevant. Now it would be a good heuristic to begin proving the "simplest" part of the theorem, namely the reflexivity of $\rho \cap \sigma$, in order to use this proof to prove the other parts "analogously". Why is this the simplest part? One might argue as a purely syntactic heuristic that the definition of the reflexivity produces only two two-literal clauses whereas symmetry produces a three-literal and two two-literal clauses, and transitivity a four-literal, a three-literal, and a two-literal clause. Consequently it is "easier" to show the reflexivity than to show the symmetry, which is itself easier to show than the transitivity, which is by far the most difficult part.

If we give the axiomatization of the definitions and of the first part of the theorem (the reflexivity) to an automated theorem prover, we might obtain the following resolution proof which the MKRP system actually produced (s_1, rel_1 and rel_2 are Skolem constants. The asterisk indicates that the following clause is used in the final proof).

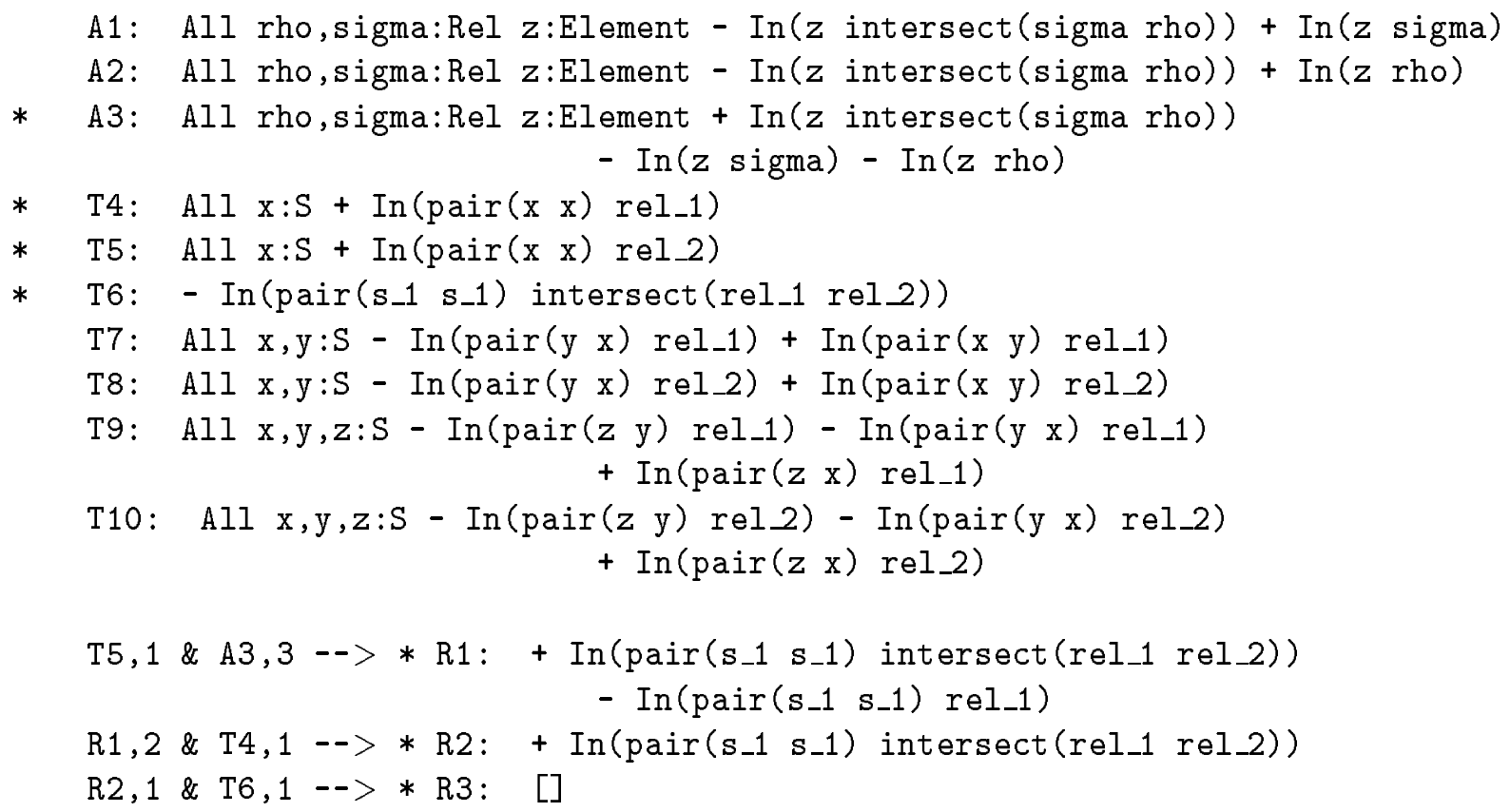

If we analyze this proof, we see that the axioms defining symmetry and transitivity have not been used, hence we can prove the reflexivity of the intersection of two equivalence relations only by using the definition of intersection and reflexivity. 
That is instead of $\forall \rho, \sigma:$ relation $P(\rho, \sigma) \Longrightarrow$ reflexive $(\rho \cap \sigma)$ we simply have to prove:

$\forall \rho, \sigma$ : relation reflexive $(\rho) \wedge$ reflexive $(\sigma) \Longrightarrow$ reflexive $(\rho \cap \sigma)$

This is easier because the original version results in a larger search space. This now is also the decisive step to notice, where we use analogy: we prove the other two parts in the "same" manner, that is, we replace "reflexive" by "symmetric" or rather "transitive". So we obtain a general proof plan for the whole proof:

- $\forall \rho, \sigma$ : relation reflexive $(\rho) \wedge$ reflexive $(\sigma) \Longrightarrow \operatorname{reflexive}(\rho \cap \sigma)$

- $\forall \rho, \sigma$ : relation symmetric $(\rho) \wedge \operatorname{symmetric}(\sigma) \Longrightarrow \operatorname{symmetric}(\rho \cap \sigma)$

- $\forall \rho, \sigma$ : relation transitive $(\rho) \wedge \operatorname{transitive}(\sigma) \Longrightarrow \operatorname{transitive}(\rho \cap \sigma)$

Abstractly speaking we use the heuristic: "Prove equal things with equal preconditions."

But analyzing the proof of the reflexivity property we observe in addition that only terms of depth one occur (no terms of the form $(\rho \cap \sigma) \cap \rho$ ). This can also be used as heuristic: "Restrict the term depth to $n$, if $n$ is the deepest nesting of terms in the proof that is used for the analogy." The use of the last heuristic is more limited, but in this case (where $n=1$ ) it works and causes a drastic improvement in the proof of transitivity. In the following table the number of resolvents in order to find the proof is shown. As one can see both heuristics together show the best results.

In the table "all" means that all three definitions of reflexivity, symmetry and transitivity are given to the theorem prover, "one" means only the necessary one is given, "unbounded" that arbitrary terms may occur, whereas in the case of "depth one" only terms of depth one may occur.

\begin{tabular}{|c||c|c|c|c|}
\hline & all \& unbounded & one \& unbounded & all \& depth one & one \& depth one \\
\hline reflexivity & 3 & 3 & 3 & 3 \\
\hline symmetry & 9 & 9 & 7 & 7 \\
\hline transitivity & 122 & 83 & 46 & 23 \\
\hline
\end{tabular}

Summarizing we see that it is possible to find powerful heuristics by using analogy. In this case a proof was possible even without domain specific heuristics, but in more difficult examples this might be no longer the case. Another interesting aspect is that analogy can be used to find the "preconditions" of a theorem (those axioms that are necessary to prove the theorem). Unlike to the workings of a human mathematician, an automated resolution theorem prover must be told all relevant facts, the so-called preconditions. The theorem prover has then to show the unsatisfiability of the facts and the negated theorem. When we started to prove the "reflexivity" we used all the definitions of all occuring concepts as preconditions. We then found a proof and analyzed it and noticed that the definitions of symmetry and transitivity have not been used. By analogy we concluded that in the other two cases too only the corresponding precondition might be necessary.

The heuristics above can also be used to prove the theorem that the intersection of two congruence relations is again a congruence relation [5, p.45], but the savings for the proof of the property that is additionally to be shown are much smaller.

But not only in a neighbourhood as close to the original theorem as these two can we use these heuristics. We can also prove for example the subgroup criterion analogously by using the heuristic "prove equal things with equal preconditions":

Define a subgroup as a subset of a group that is itself a group with respect to the same group operation. We then have the following theorem: 
Theorem: Let $(G,+)$ be a group with neutral element 0 , inverse function "_" and let $S$ be a subset of $G$. Then $(S,+)$ is subgroup of $(G,+)$ if and only if
a) $0 \in S$ and
b) $\forall x, y \in S(x+y) \in S$ and
c) $\forall x \in S(-x) \in S$

Let us consider here only the ("non-trivial") "£"-part of the proof:

We define a group by:

$$
\begin{aligned}
& \operatorname{group}(G,+, 0,-) \Longleftrightarrow 1) G \text { set } \\
& \text { 2) }+: G \times G \rightarrow G \\
& \text { 5) associative }(G,+) \\
& \text { 3) } 0 \in G \\
& \text { 4) }-: G \rightarrow G
\end{aligned}
$$

Then we can prove $\operatorname{group}(S,+, 0,-)$ by proving these conditions. Therefore we use $S \subseteq G$ in all seven cases and the corresponding facts of the precondition, i.e.

in the case of 1),5),6) and 7) no additional information,

in the case of 2) the corresponding condition b),

in the case of 3 ) condition a), and

in the case of 4 ) condition c).

As in the example above we can use here the heuristic "show equal things by equal preconditions" and reduce the used preconditions to the necessary ones.

This form of analogical reasoning can be used in the proofs of corresponding structures like rings, fields, vector spaces and so on. In all these cases the proofs can be separated into different parts, with a similiar reduction of savings in the search space.

Unfortunately this is not the whole story, since in a mathematical textbook our theorem would not be phrased as above. For example condition a) would not be mentioned, because it follows from the others. But then our analogy would not be sufficient to prove 3 ).

There are also counterexamples for the heuristic "Show equal things by equal preconditions". Let for example a group be defined by 1) through 5) above, but instead of 6) and 7) there would be axioms for the existence of a left-neutral element and the existence of left-inverses. Then it is not possible to show property 6) for the substructure using only the existence of a left-neutral element. One needs also the associativity and the existence of left-inverses. From an abstract point of view this is surprising. This proof is therefore more difficult than the other proofs above.

\section{More Examples}

In this section we want to sketch some analogies in proofs taken from [5]. All page numbers refer to [5]. A little more difficult than the proof of the previous section is the proof of the following part of a theorem (p.37):

Theorem: Let $\rho$ and $\sigma$ be two equivalence relations then the transitive hull of $\rho \cup \sigma$ (written $\left.(\rho \cup \sigma)^{t}\right)$ is also an equivalence relation. 
As in the proof of our first example in section 3 this proof can be split into the three subproofs "reflexivity", "symmetry", and "transitivity". But here "transitivity" plays a special rôle: It is possible to show that for every relation $\alpha$ the relation $\alpha^{t}$ is transitive. The proof of the other two parts are then mutually analogous:

- Prove the property for the union.

- Show the invariance of the property under closure.

The next example shows how a special case can catch the whole proof idea.

Theorem: Let $\left\{U_{i}: i \in I\right\}$ be a family of subsemi-groups of a semi-group $F$, then the intersection $\bigcap_{i \in I} U_{i}$ is also a subsemi-group of $F$, if the intersection is not empty.

The proof of this theorem (p.9) is given only for the intersection of two semi-groups. The general proof is then analogous to this special case. The analogy consists in the correspondence between " $\wedge$ " and " $\cap$ " on the one hand and " $\forall i \in I$ " and " $\bigcap_{i \in I}$ " on the other hand.

The two proofs can be sketched as:

$$
\begin{aligned}
u, v \in U \cap V & \Longrightarrow u, v \in U \wedge u, v \in V \\
& \Longrightarrow u v \in U \wedge u v \in V \\
& \Longrightarrow u v \in U \cap V
\end{aligned}
$$

$$
\begin{aligned}
u, v \in \bigcap_{i \in I} U_{i} & \Longrightarrow \forall i \in I \quad u, v \in U_{i} \\
& \Longrightarrow \forall i \in I \quad u v \in U_{i} \\
& \Longrightarrow \forall i \in I \quad u v \in \bigcap_{i \in I} U_{i}
\end{aligned}
$$

Because of its close analogy to this proof, no proof of the next theorem (p.10) is given:

Theorem: Let $\left\{U_{i}: i \in I\right\}$ be a family of subgroups of a group $F$, then the intersection $\bigcap_{i \in I} U_{i}$ is also a subgroup of $F$.

Later on an analogous theorem is formulated for families of ideals of a semi-group $F$ (p.22) and proved analogously.

It is a challenge to find similiar heuristics as shown above also for proofs that are more difficult to find. Therefore it will probably be necessary to use a hierarchical representation as proposed in [2].

\section{Analogy by an Abstract Description of Proofs}

BUNDY's idea of proof plans as introduced in [4] may also serve as a metaphor for "proving by analogy": two proofs are analogous if they are instantiations of the same proof plan. BUNDY argues that learning from proofs should be possible, that it should be possible to find a proof by using the successful proof plan of another theorem. In order to do so, it is necessary to find a basic number of structuring and description facilities that are epistemological sufficient to describe proofs and their mutual relationships in order to find analogies, even in cases that are not as easy as the previous one. Examples from the domain of Real Analysis can be found in [2], where a working system is described, that uses a higher level representation for proofs.

Let us look closer at another example: We have the following two "analogous" theorems: 
Let $A, B$ and $C$ be sets with addition + and let $\sigma$ and $\tau$ be linear functions $A \stackrel{\tau}{\longrightarrow}$ $B \stackrel{\sigma}{\longrightarrow} C$. Then $\sigma \circ \tau$ is linear.
Let $A, B$ and $C$ be topological spaces and let $\sigma$ and $\tau$ be continous functions $A \stackrel{\tau}{\longrightarrow}$ $B \stackrel{\sigma}{\longrightarrow} C$. Then $\sigma \circ \tau$ is continous.

The proofs of these theorems can be sketched as:

$$
\begin{aligned}
& \text { (1) } \tau \text { linear } \Rightarrow \forall a, a^{\prime} \in A \quad \tau\left(a+a^{\prime}\right)=\tau(a)+\tau\left(a^{\prime}\right) \\
& \text { (2) } \sigma \text { linear } \Rightarrow \forall b, b^{\prime} \in B \quad \sigma\left(b+b^{\prime}\right)=\sigma(b)+\sigma\left(b^{\prime}\right) \\
& \forall a, a^{\prime} \in A \quad \sigma \circ \tau\left(a+a^{\prime}\right) \stackrel{\text { defo }}{=} \sigma\left(\tau\left(a+a^{\prime}\right)\right) \\
& \stackrel{(1)}{=} \sigma\left(\tau(a)+\tau\left(a^{\prime}\right)\right) \\
& \stackrel{(2)}{=} \sigma(\tau(a))+\sigma\left(\tau\left(a^{\prime}\right)\right) \\
& \stackrel{\text { defo }}{=} \sigma \circ \tau(a)+\sigma \circ \tau\left(a^{\prime}\right)
\end{aligned}
$$

(1) $\tau$ cont. $\Rightarrow \forall U \subseteq B$ open $\tau^{-1}(U)$ open in $A$

(2) $\sigma$ cont. $\Rightarrow \forall V \subseteq C$ open $\sigma^{-1}(V)$ open in $B$

$(*) \forall V \subseteq C(\sigma \circ \tau)^{-1}(V) \stackrel{\text { Prop. }}{-1}^{-1} \tau^{-1} \circ \sigma^{-1}(V)$ $\stackrel{\text { defo }}{=} \tau^{-1}\left(\sigma^{-1}(V)\right)$

$$
\begin{aligned}
V \text { open in } C & \stackrel{(2)}{\Rightarrow} \sigma^{-1}(V) \text { open in } B \\
& \stackrel{(1)}{\Rightarrow} \tau^{-1}\left(\sigma^{-1}(V)\right) \text { open in } A \\
& \stackrel{(*)}{\Rightarrow}(\sigma \circ \tau)^{-1}(V) \text { open in } A
\end{aligned}
$$

$\Rightarrow \sigma \circ \tau$ continous

Abstractly speaking the proofs consist both of the following general steps in sequence, which then constitute the "proof plan":

- expand what to prove

- use definition of composition

- use the only applicable property

- use the then newly applicable property

The analogy between concepts that underly the previous example (semi-group, vector space, ... and topological spaces) resulted later on in the history of mathematics in the definition of the new concept of an "abelian category", where one can prove formally that certain proofs about rings can be generalized to arbitrary abelian categories [13, p.151].

The most important steps of the proof can be described "declaratively". The hope is that it is possible to classify the theorems and that then the proofs can be found analogously. The two theorems above would belong to a category "property inheritence in case of composition".

\section{Classification}

Because it can be rather difficult to find an analogy, it is helpful to have a classification of theorems. Taking a closer look at the first five paragraphs of [5], one can make the following rough classification of theorems (We describe only the first two categories in more detail):

- Invariance:

- 1.11: intersection of a family of subsemigroups is again a subsemigroup if it is nonempty

- 1.12: intersection of a family of subgroups is again a subgroup

- 3.3: union (intersection) of a family of ideals is again an ideal if nonempty

- 4.8.1: intersection of equivalence relations is again an equivalence relation

- 4.8.2.1: transitive closure of the union of equivalence relations is again an equivalence relation 
- Compatibility/Commutativity:

- 1.9: one of subgroup equals one of group

- 4.10.3: commutative diagram

- 4.11, 4.12: reference of equivalence relations and mappings

- Equality of Sets: 1.15, 3.9, 3.10.1-3, 3.15.1, 4.3

- Equivalences: 1.10, 2.6, 2.7, 3.6, 5.2

- Uniqueness Theorems: 2.5.1, 2.5.2, 3.10.4, 3.15.2

- Hierarchy Relation: 3.2

- Minimality: 4.8.2.2

$5.3,5.6,5.7,5.8$ are analogous to $4.8,4.10,4.11,4.12$ for congruence relations.

The theorems 5.14 and 5.17 do no fit into the categories above and an abstract description may be difficult.

For the first category one has the following description (when only two components are considered): $P(x) \wedge P(y) \wedge$ additional conditions $\Longrightarrow P(f(x, y))$

Such a classification could be useful in automated theorem proving, when one has to solve a problem, in the following way:

- at first find the corresponding category of the problem

- then find an "analogous" theorem in this category with a known proof

- try to transform the proof to the new theorem

Sometimes it should be possible to extract a proof schema for the whole category (not to find a general proof for the whole schema, but as a heuristic). Or it is at least possible to have some heuristic information as in the case of the "invariance"-category the heuristic "prove equal things with equal preconditions". Another such heuristic is Bundy's "Ripple-Out" heuristics [4, p.115].

\section{$6 \quad$ Analogy and Model}

Many mathematical fields as geometry and topology are guided by strong aspects of spatial imagination and live widely on intuition and geometrical views of things. Even in fields where one has no visual models at all, mathematicians try to use the strong visual apparatus of the human mind for an intuition of the constituting concepts and even of proofs. JACQUES HADAMARD gives an example of a "mental picture" of the proof that there are infinitely many prime numbers $[10$, p.76]. A more complex example is given by Bartel L. VAN Der Waerden in [19].

We can rephrase this by saying that mathematicians use intuitive models of the objects they deal with whenever possible. These objects are not always real models in the logical sense, more often they are only "near models" or "almost models" (a very lively discussion of this trick can be found in $[7$, p.112 f.]). The proof is searched for and visualized by means of an example. If the proof is found for the example it is generalized by finding the corresponding analogy and only then is it written down formally. 
Many parts of mathematics have been developped by abstracting and generalizing known concepts. For example topology can be regarded as a generalization dealing with open sets and continous functions on the real numbers $\mathbf{R}$. So when trying to find a general proof in topology one sometimes uses the analogous situation in the case of $\mathbf{R}$ or more often in the case of $\mathbf{R}^{2}$ to find the general proof. Let us consider a simple theorem (taken from [17, p.19f]):

We want to begin with recalling some definitions of topology: Let $(X, \mathcal{O})$ be a topological space, the members of $\mathcal{O}$ are called open sets and for all $x \in X$ let $\mathcal{U}(x)$ be the set of all surroundings of $x$, a subset $U \subseteq X$ is called a surrounding of $x$ iff there is a $V \in \mathcal{O}$ with $x \in V \subseteq U$.

Theorem: $A$ set $V \subseteq X$ is open $\Longleftrightarrow \forall x \in V \quad \exists U_{x} \in \mathcal{U}(x)$ with $U_{x} \subseteq V$

The proof:

$$
\begin{aligned}
\text { "œ": } & V \text { open } \Longrightarrow V \in \mathcal{O} \Longrightarrow \forall x \in V \quad x \in V \subseteq V \\
& \forall x \in V \quad \exists U_{x} \in \mathcal{U}(x) \text { therefore } \\
& V x=\bigcup_{x \in V}\{x\} \subseteq \mathcal{O} \text { with } x \in \bigcup_{x} \subseteq U_{x \in V} V_{x} \subseteq V \text { therefore } \\
& \text { consequently } V \text { is open as union of the open sets } V_{x} .
\end{aligned}
$$

Consider only the second (non-trivial) part of the proof. Although this proof can be found directly, it is most convenient for a mathematician to use his intuition of the plane when trying to find the proof. The set $V$ is imagined as a (finally open) circle in the plane. The $x$ 's are points inside the circle and the $U_{x}$ are small circles around the $x$ 's fully contained in $V$, the $V_{x}$ are small open circles inside the $U_{x}$ with centre $x$. This picture then induces the proof: All the small open circles $V_{x}$ form a cover of $V$.

A similiar visualization is often used to clarify the relationship between sets and their intersections, unions, or complements by so called VENN-diagrams (see [1] or another mathematical encyclopedia).

The interesting thing is that one considers only one special example in order to catch the whole proof. Pólya gives in [16] some more examples where the general case is equivalent to the special one. Such an example has to be as easy as possible (in order to be feasible) and as general as necessary (in order to include the whole problem). Now if we have a reasoner for such restricted examples one could then follow this proof of the example as a guiding yardstick in the general case. This could be done just as described above, where one proof is used to guide the search for another by analogy.

\section{Conclusion and Further Work}

We presented some examples for the possibility to find or to facilitate proofs by analogy. The examples suggest an actual formalization such that the idea can be used in an automated theorem proving system. (The examples of the last section seem to be at the moment a little far from actual formalization: this idea is appropriate only in limited cases when an intuition is helpful. The great contribution of formal logic to mathematics however has been to abstract from concrete models and to treat the problem purely formal.)

The idea is to find a whole set of representation facilities for proofs, so that the system can formulate abstract versions of proofs in order to prove other theorems by analogy. This should help in finding the preconditions of a theorem (those clauses that are actually necessary for a proof) and to automatically set certain parameters such as term depth. It also should contribute to the answer of the question "what are the main steps of the proof". 
We also notice a semantic aspect: When we draw an analogy from theorems and concepts to analogous proofs we use as a general heuristic that the proof of the generalization follows the same general steps as in the special case. One might argue that this follows from the analogy between the corresponding models. But this whole aspect is only heuristic and there are much more counterexamples than the ones mentioned above.

In this paper we have presented a proposal of how to include some aspects of the concept of "analogy" in an automated theorem prover. In order to see whether the proposed ideas are really useful it is necessary to experiment with a much larger set of theorems taken arbitrarily from a mathematical textbook. There are more problems however that have to be solved before we have a useful tool.

\section{Acknowledgements}

I would like to thank AXEL PRÄCKLEIN for thorough readings of an earlier draft and JÖRG SIEKMANN for many advices that resulted in numerous improvements.

\section{References}

[1] Heinrich Behnke, Reinhold Remmert, Hans-Georg Steiner, Horst Tietz (Edts.) (1964), "Mathematik 1" Fischer Taschenbuch Verlag, Frankfurt.

[2] Bishop Brock, Shaun Cooper, William Pierce (1988), "Analogical Reasoning and Proof Discovery" Proc. of the 9th CADE, Argonne Illinois, LNCS 310, Edts. E.Lusk \& R.Overbeek, Springer Verlag, Berlin, Heidelberg, New York.

[3] Alan Bundy (1983), "The Computer Modelling of Mathematical Reasoning" Academic Press, London.

[4] Alan Bundy (1988), "The Use of Explicit Plans to Guide Inductive Proofs" Proc. of the 9th CADE, Argonne Illinois, LNCS 310, Edts. E.Lusk \& R.Overbeek, Springer Verlag, Berlin, Heidelberg, New York.

[5] Peter Deussen (1971), "Halbgruppen und Automaten" Springer Verlag, Berlin, Heidelberg, New York.

[6] Norbert Eisinger, Hans Jürgen Ohlbach (1987), "The Markgraf Karl Refutation Procedure (MKRP)" Proc. of the 8th CADE, Oxford, England, LNCS 230, Edt. J.Siekmann, Springer Verlag, Berlin, Heidelberg, New York.

[7] Richard P. Feynman (1987), "Sie belieben wohl zu scherzen, Mr. Feynman!" Piper, München, german translation of: "Surely You're Joking, Mr. Feynman!", Norton, New York, London, 1985.

[8] T. Gergely, K.P. Vershinin (1985), "Natural Mathematical Texts vs. Programs" in Mathematical Methods of Specification and Synthesis of Software Systems '85, Proc. of the International Spring School Wendisch-Rietz, GDR, LNCS 215, Edt. W.Bibel \& K.P.Jantke, Springer Verlag, Berlin, Heidelberg, New York.

[9] Russell Greiner (1988), “Learning by Understanding Analogies" Artificial Intelligence 35 81-125.

[10] Jacques Hadamard (1945), "The Psychology of Invention in the Mathematical Field" Princeton University Press, reprinted 1954 by Dover Publication, New York. 
[11] Rogers P. Hall (1989), "Computational Approaches to Analogical Reasoning: A Comparative Analysis" Artificial Intelligence 39 39-120.

[12] Karl Mark G Raph (1984), "The Markgraf Karl Refutation Procedure" Memo-SEKI-MK-84-01, Fachbereich Informatik, Universität Kaiserslautern.

[13] Barry Mitchell (1965), “Theory of Categories" Academic Press, New York, London.

[14] Allen Newell (1981), "The Heuristic of George Polya and its Relation to Artificial Intelligence" Department of Computer Science, Carnegie-Mellon University, Pittsburgh, Pennsylvanian 15213; CMUCS-81-133, also to appear in R.Groner, M.Groner and W.F.Bishoof (Eds.), Methods of Heuristics, Hillsdale, MJ: Lawrence Erlbaum.

[15] George Pólya (1949), "How to Solve It" Princeton University Press, Princeton, New Jersey.

[16] George Pólya (1954), “Mathematics and Plausible Reasoning” Princeton University Press, Princeton, New Jersey.

[17] Boto von Querenburg (1979), "Mengentheoretische Topologie" 2.Aufl., Springer Verlag, Berlin, Heidelberg, New York.

[18] Helmut Thiele (1986), "A Model Theoretic Oriented Approach to Analogy" Proc. of the International Workshop on Analogical and Inductive Inference, Wendisch-Rietz, GDR, LNCS 265, Edt. K.P.Jantke, Springer Verlag, Berlin, Heidelberg, New York.

[19] Bartel L. van der Waerden (1964), "Wie der Beweis der Vermutung von Baudet gefunden wurde" Abh. Math. Sem. Univ. Hamburg 28. 